J. Clin. Chem. Clin. Biochem.

Vol. 27, 1989, pp. $941-946$

(C) 1989 Walter de Gruyter \& Co.

Berlin - New York

\title{
Chromogenic Endotoxin Assay in Plasma Selection of Plasma Pretreatment and Production of Standard Curves
}

\author{
By H. Fukui, Beate Brauner, J. C. Bode and Christiane Bode \\ Division of Gastroenterology, Department of Internal Medicine, Robert-Bosch-Krankenhaus, Stuttgart, F. R. G.
}

(Received August 18, 1988//February 6/August 10, 1989)

Summary: The aim of this study was to define the optimal conditions for the plasma pretreatment and to improve the production of standard curves for plasma endotoxin determination by a chromogenic substrate assay. Endotoxin standard from $E$. coli $\mathrm{O} 111$ : B $4(0-50 \mathrm{ng} / \mathrm{l})$ was added to pyrogen-free water or to plasma samples from 12 healthy subjects and 24 alcoholics, before pretreatment by heating $\left(75^{\circ} \mathrm{C}, 5\right.$ minutes $)$ or with perchloric acid $(0.32 \mathrm{~mol} / \mathrm{l})$. When endotoxin standard curves were determined using a microprocessorcontrolled reader, the slopes of the curves obtained with plasma differed from those with pyrogen-free water. The slope of the standard curve prepared with plasma samples from different patients exhibited marked interindividual variations. Compared with the heating method, the perchloric acid method gave more variable results and a lower recovery of added endotoxin, especially in plasma from alcoholics. The results permit the following conclusion:

1. For plasma endotoxin determination, a standard curve should be prepared for each individual plasma sample.

2. The endotoxin standard should be added before pretreatment of the plasma.

3. Pretreatment of the plasma by heating at $75^{\circ} \mathrm{C}$ for 5 minutes provides more reliable results than pretreatment with perchloric acid.

\section{Introduction}

Numerous studies on the determination of endotoxin in human plasma have been published $(1,2)$. Most have been performed using different variations of the classical Limulus lysate test (measurement of gel formation). The problems associated with this procedure have been discussed in detail elsewhere (3). For quantitative measurements of endotoxin in plasma, a chromogenic method hàs been described by Japanese authors (4). Although the chromogenic assay has been shown to give reliable results for minute amounts of endotoxin in water (4), measurements in blood still present difficulties. Firstly, there is still disagreement about the best way of preparing standard curves (5-8) and secondly, an optimal method for eliminating plasma inhibitors in the endotoxin assay has not yet been established. Among various pretreatment procedures aimed at avoiding inhibitory activities in plasma, dilution and heating $(9,10)$ and perchloric acid treatment (11) have been widely used. Studies comparing these methods $(11,12)$ have produced conflicting results.

The aim of this study was to define the optimal way of obtaining standard curves for a chromogenic assay and to re-evaluate plasma pretreatment procedures.

\section{Materials and Methods}

1. Subjects

Venous blood was collected from 12 healthy individuals (age range 25 to 55 years) and 24 chronic alcoholics (7 with fatty liver and 17 with liver cirrhosis, age range 60 to 65 years). The diagnosis was based on liver biopsy in all cases. 


\section{Blood sampling}

After cleaning the skin twice with aqueous ethanol, volume fraction 0.6 and with Kodan-spray (Schülke \& Mayr GmbH, Norderstedt, F. R. G.), $3 \mathrm{ml}$ blood samples were collected from the antecubital vein into pyrogen-free syringes containing 75 units of $\mathrm{NH}_{4}$-heparin as previously described (13). Care was taken to avoid air bubbles in the syringes. Platelet-rich plasma was prepared by centrifugation at $150 \mathrm{~g}$ for $20 \mathrm{~min}$ at $0^{\circ} \mathrm{C}$. The separated plasma was stored at $-80^{\circ} \mathrm{C}$ in pyrogen-free polyethylene tubes (Eppendorf, Hamburg, F. R. G.).

\section{Chromogenic assay}

Hydrolysis of the chromogenic substrate was determined in two different ways: firstly as described in the original method used (6) by incubation for three minutes and stopping the reaction with acetic acid (endpoint determination), and secondly by checking the linearity of the indicator reaction used in the test by measuring hydrolysis kinetics of the chromogenic substrate (continuous assay).

\subsection{Endpoint determination}

Endotoxin was measured in plasma by a modification of the chromogenic substrate test (Kabi-Vitrum, Stockholm, Sweden) as described by Friberger et al. (6). The plasma was diluted 1:10 with ice-cold pyrogen-free water. In order to prepare a large number of samples for parallel incubation, the diluted plasma samples were allowed to stand for a maximum of 30 minutes on ice. Storage of these plasma samples, diluted 1:10 on ice, resulted in no loss of endogenous or added endotoxin over a period of up to two hours. The diluted plasma samples were then heated for $5 \mathrm{~min}$ at $75^{\circ} \mathrm{C}$. Fifty $\mu \mathrm{l}$ of Limulus amoebocyte lysate was added to $50 \mu \mathrm{l}$ of the heated plasma. After a preincubation time of $25 \mathrm{~min}\left(37^{\circ} \mathrm{C}\right), 100 \mu \mathrm{l}$ of substrate (S2423, Ac-Ile-Glu-Gly-Arg-p-nitroanilide, $1.4 \mathrm{mmol} / \mathrm{l}$ ) dissolved in Tris buffer ( $25 \mathrm{mmol} / \mathrm{l}, \mathrm{pH} 9.0)$ were added and incubated at $37^{\circ} \mathrm{C}$ for exactly $3 \mathrm{~min}$. The reaction was stopped by adding $100 \mu \mathrm{l} 3.3 \mathrm{~mol} / \mathrm{l}$ acetic acid. Absorbance was measured at 405 $\mathrm{nm}$. The test was performed in sterile polyethylene tubes. All procedures were performed in a laminar flow bank (Captair 2004, Hermann Waldner KG, Wangen im Allgäu, F. R. G.). In accordance with the instructions provided by the manufacturer all determinations were performed in duplicate.

Two blank values were run in parallel with each test: one in which the same volume of pyrogen-free water was added instead of $50 \mu$ l diluted plasma, and a second with which the intrinsic absorbance of the plasma was to be measured, and in which Limulus amoebocyte lysate was replaced by $50 \mu$ l pyrogen-free water. Otherwise, the two blank values were treated in the same way as the plasma measurement values.

\subsection{Continuous measurement}

The kinetics of the enzymatic hydrolysis of the chromogenic substrate were measured at $37^{\circ} \mathrm{C}$ for $0-10 \mathrm{~min}$, using sterile microtitre plates in a micro-processor controlled reader (EAR400AT, SLT Lab. Inst., Salzburg, Austria), which was placed in a laminar flow bank. Preparation of the plasma samples (including preincubation and addition of substrate) was exactly as described under 'endpoint determination', with the sole exception that the volumes were reduced to $40 \mu \mathrm{l}$ sample, $40 \mu \mathrm{l}$ Limulus amoebocyte lysate and $80 \mu \mathrm{l}$ substrate buffer. Measurement of the change in the absorbance was carried out at intervals of 30 seconds at $405 \mathrm{~nm}$.

\section{Standard curves and calculation of endogenous endotoxin}

Endotoxin standards were prepared from $E$. coli O 111:B 4 (Kabi Vitrum, Stockholm, Sweden; $1 \mathrm{ng}=12$ endotoxin units (EU). According to the information of the manufacturer the endotoxin unit (EU) is determined against the EC5 standard of the Food and Drug Administration (FDA) or lot F, USP reference standard endotoxin and controlled under FDA licence No 709. To each $100 \mu \mathrm{l} 1: 5$ diluted pláśma sample, $100 \mu \mathrm{l}$ of pyrogen-free water or $100 \mu \mathrm{l}$ of different standard endotoxin solutions $(12.5-100 \mathrm{ng} / \mathrm{l})$ were added before heating. This gave final endotoxin concentrations of $0,6.25,12.5,25,37.5$ and 50 $\mathrm{ng} / \mathrm{l}$. After adding the respective amount of standard endotoxin solution, the samples were ice-cooled for a maximum of five minutes before heating.

To plot the standard curves, the measured changes in absorbance were used for each standard value after subtracting the value for the plasma sample (with no addition of exogenous endotoxin standard).

The endogenous endotoxin concentration was calculated as follows:

Endotoxin $\left(10^{3} \mathrm{EU} / \mathrm{l}\right)=$

$\left[\mathrm{A}_{\mathrm{pl}}-\mathrm{A}_{\mathrm{H}_{2} \mathrm{O}}-\mathrm{A}_{\mathrm{pl}}\right.$ blank $] \times \frac{0.2 \mathrm{EU} / \mathrm{ml}-0.1 \mathrm{EU} / \mathrm{ml}}{\Delta \mathrm{A}_{0.2 \mathrm{EU}}-\Delta \mathrm{A}_{0.1 \mathrm{EU}}} \times 10$

where

$\mathrm{A}_{\mathrm{pl}}=$ absorbance for plasma

$\mathrm{A}_{\mathrm{H}_{2} \mathrm{O}}=$ absorbance for water (both after incubation with Limulus amoebocyte lysate)

$\mathrm{A}_{\mathrm{pl}}$ blank = absorbance for plasma blank

$\Delta \mathrm{A}_{0.2 \mathrm{EU}}$ and $\Delta \mathrm{A}_{0.1 \mathrm{EU}}$ were read off the standard curve.

Fraction bar $=$ reciprocal value of the slope of the standard curve.

\section{Recovery}

In order to establish how much exogenous endotoxin is lost by heating, another endotoxin standard with a final concentration of $25 \mathrm{ng}$ endotoxin per litre $1: 10$ diluted plasma was prepared by adding endotoxin to each plasma after heating. The increase in absorbance in the samples with added endotoxin after heating was taken as $100 \%$, and the recovery of the endotoxin added to the sample before heating was calculated as the recovered percentage as follows:

Recovery $\%=\mathrm{A} / \mathrm{B} \times 100$,

where

$A=$ net increase in absorbance in the sample with endotoxin added before treatment,

B = net increase in absorbance in the sample with endotoxin added after treatment. In each run of the assay, a standard curve in pyrogen-free water was also prepared as a control.

\section{Acid treatment}

In order to compare the results obtained with pretreatment of the plasma by heating as described in section 3.1 with those obtained with the method proposed by Obayashi (11) in which plasma is treated with acid before endotoxin determination, two variations of the test with acid pretreatment were performed as follows.

6.1 Plasma samples from 22 subjects ( 8 healthy individuals, 7 alcoholics with fatty liver and 7 alcoholics with liver cirrhosis) were treated using the original method of Obayashi (11). Plasma $(180 \mu \mathrm{l})$ was mixed with either $20 \mu \mathrm{l}$ pyrogen-free water or endotoxin standard solution at a concentration of $0-50 \mathrm{ng}$ exogenous endotoxin per litre of diluted $(3: 20)$ plasma.

$\therefore$

J. Clin. Chem. Clin. Biochem. / Vol. 27, 1989 / No. 12 
6.2 It was considered that recovery might be better if the plasma was diluted further. Plasma samples $(100 \mu \mathrm{l})$ from the 22 subjects mentioned in 6.1 were mixed with $100 \mu$ l water or endotoxin standard solution at an exogenous endotoxin concentration of $0-50 \mathrm{ng}$ per litre of diluted $(1: 12)$ plasma.

The diluted plasma samples $(200 \mu \mathrm{l})$ pretreated as described under 6.1 and 6.2 were mixed with $400 \mu \mathrm{l}$ of $0.32 \mathrm{~mol} / 1 \mathrm{HClO}_{4}$, incubated for $20 \mathrm{~min}$ at $37^{\circ} \mathrm{C}$ and centrifuged at $1000 \mathrm{~g}$ for 15 $\min$ at $0^{\circ} \mathrm{C}$. An aliquot $(100 \mu \mathrm{l})$ of each supernatant was neutralized by adding of $100 \mu \mathrm{l} 0.18 \mathrm{~mol} / \mathrm{l} \mathrm{NaOH}$; tests on 6 plasma samples showed that this treatment resulted in a pH of 7-7.5. Final plasma dilutions were therefore $3: 20$ and $1: 12$ respectively. Another endotoxin standard with a final concentration of $25 \mathrm{ng}$ endotoxin per litre plasma (diluted 3:20 or $1: 12)$ was prepared by adding endotoxin to the neutralized supernatant. The recovery rate of added endotoxin was calculated as described in section 4 . For each run of the assay, endotoxin standard $(25 \mathrm{ng} / \mathrm{l})$ was prepared in pyrogen-free water both with and without $\mathrm{HClO}_{4}$ pretreatment.

\section{Self-prepared control samples}

To evaluate the variation in endotoxin concentration over time from one determination to the next, plasma from a healthy individual which was both spiked and non-spiked with exogenous endotoxin $(25 \mathrm{ng} / \mathrm{l})$ was divided into $0.5 \mathrm{ml}$ portions in sterile polyethylene tubes and stored at $-80^{\circ} \mathrm{C}$. The above sample was measured weekly for 1 month and thereafter in parallel with every endotoxin determination of the study over a period of 8 months to obtain a measure of the precision of the assay and the stability of endotoxin during storage.

\section{Statistical analysis}

For statistical evaluation, Wilcoxon-Mann-Whitney's U-test was used. The results were expressed as means $\pm \mathrm{SD}$.

\section{Results}

\section{Standard curves}

The kinetics of the hydrolysis of the chromogenic substrate with endotoxin standards in water and in plasma from healthy individuals (fig. 1) was linear for $3 \mathrm{~min}$. The endogenous endotoxin concentrations in the plasma from the healthy individuals never exceeded 60 endotoxin units per litre $(=5 \mathrm{ng} / \mathrm{l})$. The net increase in the absorbance $(\Delta \mathrm{A})$ obtained in the plasma samples was 4 to 5 times greater than that observed in water. When endotoxin standards were added to plasma from two patients with very high endogenous endotoxin concentrations ( $>2400$ endotoxin units per litre $=>200 \mathrm{ng} / \mathrm{l}$ ), deviations from linearity were seen after 2 and $2.5 \mathrm{~min}$, respectively.

The slope of the standard curve in each plasma exhibited marked interindividual variation, particularly in the alcoholics (fig. 2). Each standard curve was linear up to $37.5 \mathrm{ng}$ endotoxin per litre of diluted $(1: 10)$ plasma (corresponding to a final concentration of $375 \mathrm{ng} / \mathrm{l}=4500$ endotoxin units per litre of plasma; fig. 2).

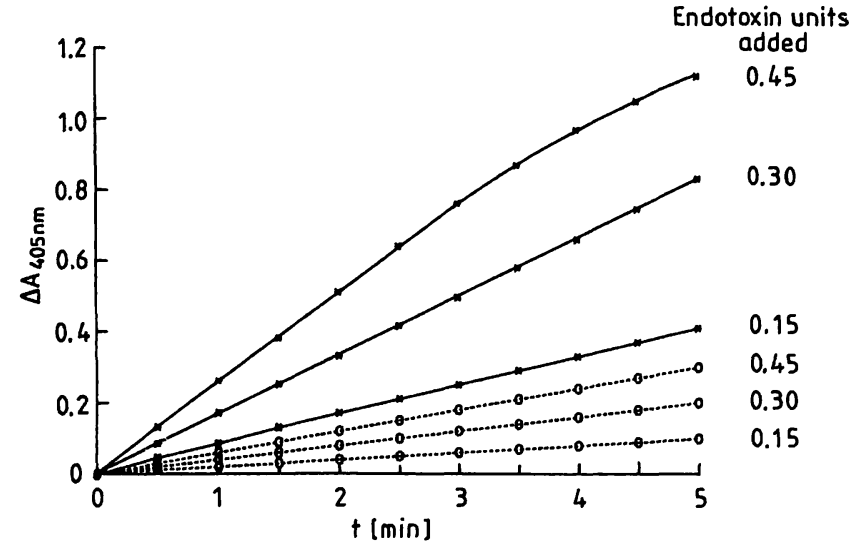

Fig. 1. Kinetics of the hydrolysis of the chromogenic substrate by endotoxin standards in distilled pyrogen-free water $(0--0)$ and in plasma $(x-x)$ from a healthy subject, using microtitre plates. The net increase in absorbance $\left(\Delta \mathrm{A}_{405 \mathrm{~mm}}\right)$ was obtained by subtracting the value of water or plasma without endotoxin. The assay was performed in $40 \mu$ samples.
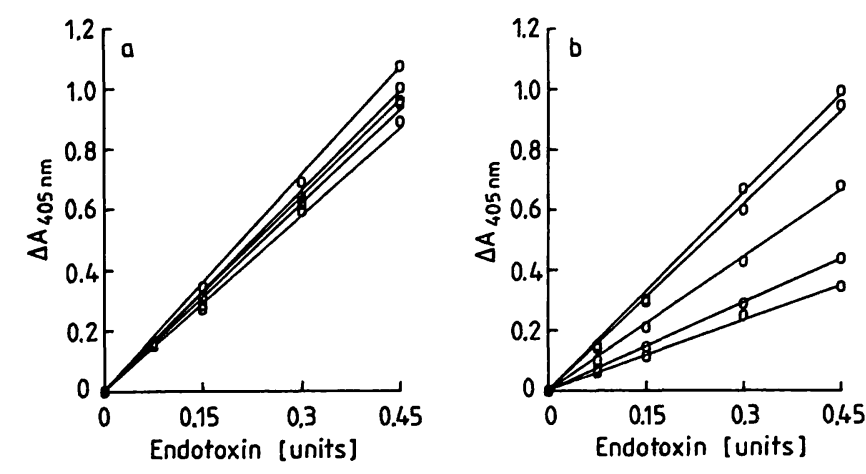

Fig. 2. Standard curves of endotoxin in plasma samples of 5 healthy subjects (a) and 5 patients with alcoholic liver disease (b). Determinations were performed using the endpoint determination (sample volume: $50 \mu \mathrm{l}$ ). Note the marked variation of the slope of the standard curves in the group with alcoholic liver disease.

Linearity was also observed up to $3 \mathrm{~min}$ for samples with endotoxin concentrations up to $50 \mathrm{ng}$ per litre diluted $(1: 10)$ plasma when the hydrolysis of the chromogenic substrate was measured continuously (fig. 1). For the two plasma samples containing very high endogenous endotoxin concentrations, the linear parts of the kinetic curves were extrapolated to $3 \mathrm{~min}$ and used to draw standard curves.

\section{Recovery of endotoxin by the heating method}

The recovery of added endotoxin was $91.3 \pm 13.3 \%$ (mean \pm SD) in plasma from controls (fig. 3), and $89.8 \pm 15.1 \%$ in plasma from the total group of alcoholics. The recovery rate in plasma from cirrhotics $(87.4 \pm 17.3 \%)$ was not significantly different from that in plasma from patients with fatty liver $(95.7$ $\pm 4.4 \%)$. 


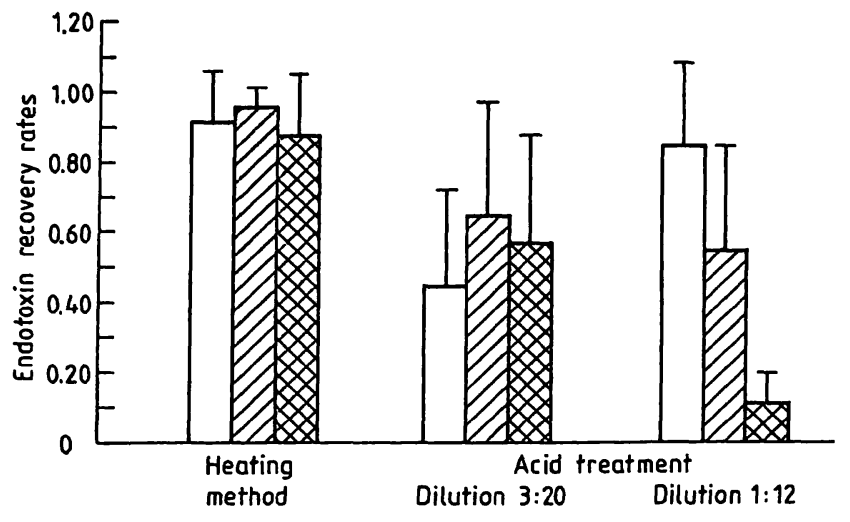

Fig. 3. Comparison of the three different procedures for eliminating factors interfering with the endotoxin determination using a chromogenic substrate. The recovery rates of endotoxin standard with these procedures were compared in plasma samples obtained by the heating method from 12 healthy subjects (normal), 7 patients with alcoholic fatty liver and 17 patients with alcoholic cirrhosis.

For both methods using perchloric acid, the number of subjects in the three troups was 8,7 and 7 , respectively. $\square$ normal $\square$ fatty liver $\square$ cirrhosis

3. Recovery of endotoxin by the $\mathrm{HClO}_{4}$ method

The recovery of added endotoxin was highly variable, both in plasma from controls $(44.3 \pm 27.0 \%)$ and in plasma from alcoholics with cirrhosis $(58.5 \pm 29.5 \%)$ when the acid method 1 (final plasma dilution $3: 20$ ) was used. There was no significant difference in the recovery rates between healthy subjects and the two groups with alcoholic liver disease (fig. 3). The recovery rates of endotoxin in plasma both from controls and alcoholics with this method were significantly lower $(P<0.005, P<0.01)$ than those obtained with the heating method.

The recovery of added endotoxin from plasma samples of controls was $84.3 \pm 22.9 \%$ when acid method 2 (final plasma dilution $1: 12$ ) was used. Using this method, the recovery of endotoxin from plasma of alcoholics with fatty liver varied greatly (57.9 $\pm 27.6 \%)$ and was lower than in healthy subjects $(\mathrm{P}<0.05)$. The lowest recovery rate was noted in cirrhotics $(11.6 \pm 7.5 \%)$. The recovery rates of endotoxin in plasma from patients with fatty liver and from cirrhotics by this method were significantly lower than those obtained with the heating method $(\mathrm{P}<0.025, \mathrm{P}<0.005)$ (fig. 3).

\section{The endotoxin standard in water}

Endotoxin standards $(25 \mathrm{ng} / \mathrm{l})$ in pyrogen-free water, always gave lower absorbance values than those in the plasma samples with addition of endotoxin added before heating (fig. 1).

\section{Self-prepared control samples}

As shown in figure 4, the values of the self-prepared control samples remained fairly constant for a period of almost 8 months. The mean value $\pm S D$ of all determinations of the self-prepared control samples was $300 \pm 45$ endotoxin units per litre $(n=22)$. The results obtained by the endpoint method $(\overline{\mathrm{x}} \pm \mathrm{SD}$ $=290 \pm 26$ endotoxin units per litre; $n=10$ ) were comparable to those obtained by the continuous measurement using microtitre plates $(\overline{\mathrm{x}} \pm \mathrm{SD}=312$ \pm 56 endotoxin units per litre; $n=12$ ). The coefficient of variation between days of determination was $15 \%$ for all values $(n=22)$. Related to the periods when only the "endpoint" or the "continuous" method was used, the variation coefficient was $8.9 \%$ $(n=10)$ and $18.1 \%(n=12)$ respectively.

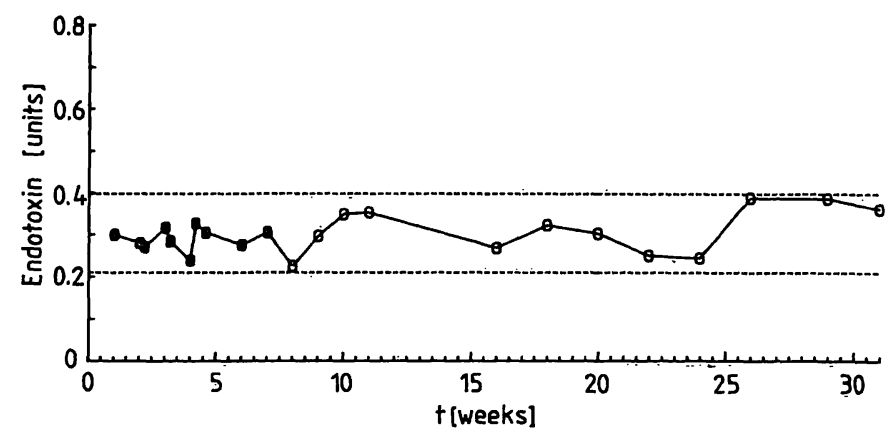

Fig. 4. Repeated determinations of endotoxin in the self-prepared control samples. The first 10 determinations were performed using the endpoint determination (solid circles), the following 12 determinations using the continuous assay (open circles). The dotted line represents the means $\pm 2 \mathrm{SD}$ of all determinations.

\section{Discussion}

After a sensitive chromogenic substrate assay had been developed for the determination of endotoxin concentration in blood, several authors $(5,7,10,12$, 14) tried to establish the optimal assay conditions. A number of important points, such as heparin concentration in plasma, inactivation temperature for inhibitors, assay $\mathrm{pH}$, influence of $\mathrm{Ca}^{2+}, \mathrm{Mg}^{2+}$ or $\mathrm{Na}^{+}$, stability of lysate and endotoxin standard, recovery of added endotoxin from platelet-rich and plateletpoor plasma have already been exhaustively investigated $(5,7,10,12,15)$. However, the questions of how to establish the standard curve for calculating plasma endotoxin concentration and how to pretreat the plasma sample adequately to minimize binding or inactivation of endotoxin have not yet been satisfactorily resolved $(8,15-19)$. The differences in the methods used to pretreat plasma and establish standard curves might influence the results of plasma endotoxin determinations in patients with various diseases. 
In previous studies on the determination of endotoxin in the plasma of patients with various diseases, standard curves of endotoxin were prepared either in pyrogen-free water $(11,15,19)$ or in pooled plasma from healthy subjects (7). Our own results show that the kinetics of the hydrolysis of the chromogenic substrate in plasma differs from that in pyrogen-free water. The net increase in absorbance of the plasma was 4 to 5 times greater than that of water. Harris et al. (12) also reported higher absorbance readings with dilutions of endotoxin in plasma compared with similar dilutions in water, and supposed that a physicochemical change of endotoxin in plasma might lead to enhanced reactivity. On the basis of these results, we suggest that standard curves in water should not be used for the calculation of endotoxin concentration in plasma. The present study further reveals that the standard curve in each plasma sample exhibits marked interindividual variation. Since the slope of the standard curve varies greatly, endotoxin plasma concentration in each patient must be calculated from its own individual standard curve. Calculations performed using a common standard curve prepared for pooled plasma will frequently lead to false results. Urbaschek et al. (20) also emphasized this point on the basis of their results in a densitometric kinetic Limulus lysate microtitre test.

In the present study, we have been able to show that the mean recovery rate of exogenous endotoxin with the heating method was about $90 \%$ and generally satisfactory both in control plasma and in plasma from patients. But the fact that the recovery of exogenous endotoxin added to the plasma before heating was usually less than $100 \%$ supports the assumption that the spiking of plasma should be done in samples both before and after heating. This procedure permits the calculation of the amount of endogenous endotoxin lost by heating, and a more accurate calculation of the endotoxin concentration in a given plasma. The problem mentioned above, namely that the percentage of endotoxin, bound or inactivated at the time of blood sampling is not measured $(8,15-$ 19), is, however, not resolved in this way.

An endotoxin assay with an individual standard curve for each plasma certainly improves reliability, but makes the endotoxin determination more expensive. It is therefore reasonable to attempt to reduce sample and lysate volume to minimize costs. Using the endpoint assay in plastic tubes, we obtained satisfactory results with $50 \mu 1$ of samples and lysates. When microtitre plates were used for the continuous assay a further volume reduction to $40 \mu \mathrm{l}$ was achieved. A similar reduction in the volume of plasma and lysate has been obtained by others (14).
In the present study, the recovery rates of endotoxin from spiked samples were lower with the $\mathrm{HClO}_{4}$ method than with the heating method. In contrast to our study, other authors $(11,19)$ have reported a recovery of endotoxin with the $\mathrm{HClO}_{4}$ method of almost $100 \%$.

The reason for the discrepancy between this figure and our own results is difficult to explain. One factor which might contribute to the high endotoxin recovery by Obayashi (11) and Yajima et al. (19) is the fact that these authors divided the net increase of absorbance in plasma by that obtained in water to calculate their recovery rates. We have demonstrated that, for a given amount of exogenous endotoxin, the increase in absorbance is higher in neutralized supernatant from plasma treated with $\mathrm{HClO}_{4}$ than in the same volume of water. With their mode of calculation Obayashi (11) and Yajima et al. (19) might have overestimated the percentage recovery rate. It has been generally accepted that endotoxin activities of lipopolysaccharides, including Limulus amoebocyte lysate gelating activity, are located in their lipid A moiety $(21,22)$. Acid hydrolysis has been widely used to isolate lipid A from various lipopolysaccharides (2325). Therefore, $\mathrm{HClO}_{4}$ treatment might split at least a part of endogenous and exogenous endotoxin, leading to the formation of free lipid $\mathrm{A}$, which would then be found in the precipitate after centrifugation. In addition endotoxin bound to protein might also precipitate. This precipitated part of endotoxin activity is likely to be missed by the $\mathrm{HClO}_{4}$ method.

The observed difference in recovery rates for the diluted $(3: 20)$ plasma as compared with the diluted (1:12) plasma, using the $\mathrm{HClO}_{4}$ method, supports the assumption that recovery of endotoxin in the supernatant depends on the amount of protein or other compounds that can bind or inactivate endotoxin in the acidified mixtures.

The results of the determination in the self-prepared control samples further support the assumption that the method described for endotoxin determination in plasma has an acceptable level of reproducibility. In addition, they show that under the conditions of our study the endotoxin concentration in plasma remains nearly unchanged for a period of eight months.

\section{Acknowledgement}

We thank Kabi-Vitrum for supporting our study by supplying COATEST-Endotoxin. 


\section{References}

1. Nolan, J. P. (1981) Endotoxin, reticuloendothelial function, and liver injury. Hepatology $1,458-465$.

2. Sturk, A. \& ten Cate, J. W. (1985) Endotoxin testing revisited. J. Clin. Microbiol. 4, 382-385.

3. Levin, J. (1982) The Limulus test and bacterial endotoxins: some perspectives. In: Endotoxins and their detection with the Limulus amebocyte lysate test (Watson, S., Levin, J. \& Novitsky, T. J., eds.) pp. 7-24 New York: A. R. Liss.

4. Iwanaga, S., Morita, T. \& Harada, T. (1978) Chromatogenic substrates for horseshoe crab clotting enzyme: it's application for the assay of bacterial endotoxins. Hemostasis 7, 183-188.

5. Sturk, A., Joop, K., ten Cate, J. W. \& Thomas, L. L. M. (1985) Optimalization of a chromogenic assay for endotoxin in blood. In: Bacterial Endotoxins: structure, biomedical significance and detection with Limulus amebocyte lysate. test (ten Cate, J. W., Büller, H. R., Sturk, A. \& Levin, J., eds.) pp. 117-136 New York: A. R. Liss.

6. Friberger, P. (1985) The design of a reliable endotoxin test. In: Bacterial endotoxins: structure, biomedical significance and detection with Limulus amebocyte lysate test (ten Cate, J. W., Büller, H. R., Stark, A. \& Levin, J., eds.) pp. 139149 New York: A. R. Liss.

7. Sturk, A., Janssen, M. E., Muylaert, F. R., Joop, K., Thomas, L. L. M. \& ten Cate, J. W. (1987) Endotoxin testing in blood. Progr. Clin. Biol. Res. 231, 371-385.

8. Piotrowics, B. I., Edlin, S. E. \& McCartney, A. Ch. (1985) A sensitive chromogenic Limulus amoebocyte lysate microassay for detection of endotoxin in human plasma and in water. Zbl. Bakt. Hyg. A260, 108-112.

9. Cooperstock, M. S., Tucker, R. P. \& Baublis, J. V. (1975) Possible pathogenic role of endotoxin in Reyes syndrome. Lancet $I, 1272-1274$

10. Friberger, P., Knös, M. \& Mellstam, L. (1982) A quantitative endotoxin assay utilizing LAL and a chromogenic substrate. In: Endotoxin and their detection with the Limulus amebocyte lysate test (Watson, S., Levin, J. \& Novitsky, T. J., eds.) pp. 195-206 New York: A. R. Liss.

11. Obayashi, T. (1984) Addition of perchloric acid to blood samples for colorimetric limulus test using chromogenic substrate: comparison with conventional procedures and clinical application. J. Lab. Clin. Med. 104, 321-330.

12. Harris, R. I., Stone, P. C. W. \& Stuart, J. (1983) An improved chromogenic substrate endotoxin assay for clinical use. J. Clin. Pathol. 36, 1145-1149.

13. Bode, C., Kugler, V. \& Bode, J. C. (1987) Endotoxemia in patients with alcoholic and non-alcoholic cirrhosis and in subjects with no evidence of chronic liver disease following acute alcohol excess. J. Hepatology 4, 8-14.

14. Scully, M. F., Newman, Y. M., Clark, S. E. \& Kakkar, V. V. (1980) Evalution of a chromogenic method for endotoxin measurement. Thromb. Res. 20, 263-270.
15. Tachiyama, G., Sakon, M., Kambayashi, J., Ohshiro, T. \& Mori, T. (1986) Chromogenic assay of endotoxin in platelet poor or rich plasma. Thromb. Res. 37, 309-317.

16. Pearson, F. C., Dubczak, J., Weary, M., Bruszer, G. \& Donohue, G. (1985) Detection of endotoxin in plasma of patients with gram-negative bacterial sepsis by the Limulus amoebocyte lysate assay. J. Clin. Microbiol. 21, 865-868.

17. Bigatello, L. M., Broitman, S. A.,'Fattori, L., Di Paoli, M., Pontello, M., Bevilacqua, G. \& Nespoli, A. (1987) Endotoxemia, encephalopathy, and mortality in cirrhotic patients. Am. J. Gastroenterol $8211-15$.

18. Büller, H. R., ten Cate, J. W., Sturk, A. \& Thomas, L. L. (1985) Validity of the endotoxin assay in post surgical patients. In: Bacterial endotoxins: structure, biomedical significance and detection with Limulus amoebocyte lysate test (ten Cate, J. W., Büller, H. R., Sturk, A. \& Levin, J., eds.) pp. 405-416 New York: A. R. Liss.

19. Yajima, Y., Fukuda, I., Otsuki, M., Suzuki, H., Ota, S., Ishii, M., Mori, K. \& Goto, Y. (1985) Endotoxemia in liver diseases: detection by a quantitative assay using chromogenic substrate with perchloric acid pretreatment. Tohoku J. Exp. Med. 147, $411-419$.

20. Urbaschek, B., Becker, K. P., Ditter, B. \& Urbaschek, R. (1985) Quantification of endotoxin and sample-related interferences in human plasma and cerebrospinal fluid using a kinetic Limulus amoebocyte lysate microtiter test. In: Microbiology (Leive, L., ed.) pp. 39-43. Washington DC, American Society for Microbiology.

21. Galanos, C., Lüderitz, O., Rietschel, E. Th. \& Westphal, O. (1977) Newer aspects of the chemistry and biology of bacterial lipopolysaccharides with special reference to their lipid A component. In: International Review of Biochemistry (Goodwin, T., ed.) Vol. 14: Biochemistry of Lipids II. pp. 239-335 Baltimore, University Park Press.

22. Rietschel, E. Th., Galanos, C., Lüderitz, O. \& Westphal, O. (1982) Chemical structure, physiological function and biological activity of lipopolysaccharides and lipid A. In: Immunopharmacology and regulation of leukocyte function (Webb, D. R., ed.) pp. 183-229 New York, Marcel Dekker.

23. Galanos, C., Lüderitz, O. \& Westphal, O. (1971) Preparation and properties of antisera against the lipid A component of bacterial lipopolysaccharides. Eur. J. Biochem. 24, 116-122.

24. Galanos, C. \& Lüderitz, O. (1975) Electrodialysis of lipopolysaccharides and their conversion to uniform salt forms. Eur. J. Biochem. 54, 603-610.

25. Wollenweber, H. W., Seydel, U., Lindner, B., Lüderitz, O. $\&$ Rietschel, E. Th. (1984) Nature and location of amidebound (R)-3-acyloxyacyl groups in lipid A of lipopolysaccharides from various gram-negative bacteria. Eur. J. Biochem. 145, 256-272.

Christiane Bode, Ph. D.
Research Laboratory
Department of Internal Medicine
(Gastroenterology)
Robert-Bosch-Krankenhaus
Auerbachstr. 110
D-7000 Stuttgart 50

